\title{
GEOTECNOLOGIAS APLICADAS À AVALIAÇÃO DE TAXAS DE IMPERMEABILIZAÇÃO DA TERRA EM AMBIENTE URBANO
}

\author{
Filipe da Silva Peixoto ${ }^{1}$ \\ Itabaraci Nazareno Cavalcante ${ }^{2}$ \\ Michael Vandesteen Silva Souto ${ }^{3}$ \\ Robert de Lima Muniz ${ }^{4}$ \\ José Alexandre Berto de Almada ${ }^{5}$
}

\begin{abstract}
Resumo: Este estudo buscou avaliar a taxa de ocupação e impermeabilização da terra em sub-bacias hidrográficas urbanas no município de Fortaleza - CE. Para isso, foi realizada aplicação do método de classificação de imagem orientada ao objeto, conhecido como Geobject Based In Information Analysis (GEOBIA), em uma imagem do satélite Quickbird-2 com 0,6 m de resolução espacial. Os dados foram expressos em percentuais e agregados espacialmente por meio de polígonos correspondentes aos setores censitários da área. Essas taxas foram comparadas aos limites de impermeabilização dos lotes estabelecidos pela Lei Municipal no 7.987/96. De acordo com as informações produzidas, a classificação foi avaliada com 78,9 do coeficiente de Kappa como 78,7 considerado de conformidade substancial. Dos 93 setores censitários analisados, constatou-se que 22 estão fora dos padrões, apresentando taxas de impermeabilização maiores $60 \%$, alguns setores possuem $85 \%$ de sua área impermeabilizada. A metodologia aplicada contribui para o controle da impermeabilização e fiscalização do solo urbano, além de ser realizada com rapidez de produção de informações e subsídios para gestão urbana.
\end{abstract}

Palavras-chave: Uso e cobertura da terra, Informação baseada em análise de Geobjetos, Planejamento urbano.

\section{GEOTECHNOLOGIES APPLIED TO EVALUATION OF LAND IMPERMEABILIZATION RATES}

\footnotetext{
${ }^{1}$ Doutor em Geologia. Professor do Departamento de Geografia, Universidade do Estado do Rio Grande do Norte, Campus central-UERN. Email:felipepeixoto@uern.br

2 Doutor em Geociências. Professor do Departamento de Geologia, Universidade Federal do Ceará, Campus do Pici. DGEO/UFC. Email:itabaracicavalcante@gmail.com

${ }^{3}$ Doutor em Geociência e Geofísica. Professor do Departamento de Geologia, Universidade Federal do Rio Grande do Norte - UFRN. Email:michael.souto@gmail.com

${ }^{4}$ Mestre em Geociências. Programa de Pós-graduação em Geociências, Universidade Estadual de Campinas - UNICAMP. Email: robert.muniz.geo@gmail.com

${ }^{5}$ Doutor em Geografia. Professor do Departamento de Geografia, Universidade do Estado do Rio Grande do Norte, Campus central-UERN.Email: josealmada@uern.br
} 
Abstract: The study aimed to evaluate soil impermeabilization in urbans watersheds in Fortaleza - CE. For this, we realized Geobject Based in Information Analysis (GEOBIA) application in Quickbird-2 satellite image, with $0,6 \mathrm{~m}$ of the spatial resolution. We aggregate the data to census sector, producing impermeabilization rate to each sector. We compared this rate to limits established by Municipal Law no 7.987/96. The classification was evaluated with 78,7 of Kappa's coefficient, considered a substantial confided. 93 census sectors were analyzed, and we observed that 22 census sectors showed soil impermeabilization rates above $60 \%$, some sectors have been $85 \%$ of impermeabilized rate. The applied methodology contributes to impermeabilization control end inspection of the land use, mainly it can be realized more speedily that conventional control forms.

Keyword: Use land, Geobject Based in Information Analysis, Urban planning.

\section{INTRODUÇÃO}

O controle do uso e cobertura da terra é uma das condições bases para manutenção da drenagem urbana como um componente do saneamento básico. Os serviços de drenagem urbana do Brasil se debruçam sobre os problemas das inundações, muitas vezes desarticuladas das causas dessas, como excessiva taxa de impermeabilização da terra, ocupação de áreas ribeirinhas e de alta fragilidade de determinados ambientes, priorizando medidas estruturais aplicadas a custosas obras de contenção de enchentes. As leis orgânicas de uso do solo, possuem uma função fundamental para o controle das inundações urbanas, porém a falta de fiscalização e efetividade dessas leis provoca uma espécie de retroalimentação onde lotes urbanos ultrapassam alguma medida restritiva provocando impactos sobre 0 escoamento superficial que aumenta a demanda pelo serviço de drenagem, a cargo de financiamentos públicos. Mattes (2005) explica que em função disso o financiamento das obras de drenagem via fundos públicos ou empréstimos de bancos internacionais beneficia o causador do problema.

O estudo se debruçou sobre um período em que a cidade de Fortaleza possuía um único plano que se referia ao uso e cobertura da terra, instituído pela lei orgânica de ํo 7.987/96, mas que começou de fato a ser implantada em 2006. Um importante aspecto do plano foi determinar a taxas máximas de ocupação por lote, procurando, sobretudo, reduzir a impermeabilização do solo, porém, não previu a criação e articulação de mecanismos e pessoal para fiscalização dessa natureza, e medidas punitivas.

A área de estudo trata-se de quatro sub-bacias hidrográficas urbanas contínuas, que são drenadas para o rio Coaçú, inserido na Bacia Hidrográfica do rio Cocó (Figura 1), no estado do Ceará. Através de técnicas de georreferenciamento de imagem, segmentação e classificação, e integralização e correção das classes em ambiente de Sistema de Informação Geográfica - SIG foi possível quantificar a impermeabilização local, causada pelo processo de urbanização. $A$ área de estudo está inserida na Secretaria Regional VI, abrangendo total ou parcialmente os bairros de Messejana, Curió, Guajerú, José de Alencar, Cambeba, Sapiranga, Lagoa Redonda e Cidade dos Funcionários. A área total avaliada é de $20,9 \mathrm{~km}^{2}$, que é drenada por córregos que deságuam em lagoas costeiras, representadas pelas lagoas de Messejana e da Sapiranga.

A área estudada, se situa na periferia da cidade de Fortaleza, e está em consoante processo de expansão urbana desde o início dos anos 2000, porém 
grande parte da área não dispõe de obras para controle de drenagem e possui serviço de coleta de esgoto em apenas 16\% dos domicílios (PEIXOTO et al., 2017).

Figura 1. Mapa de Localização da área de estudo.

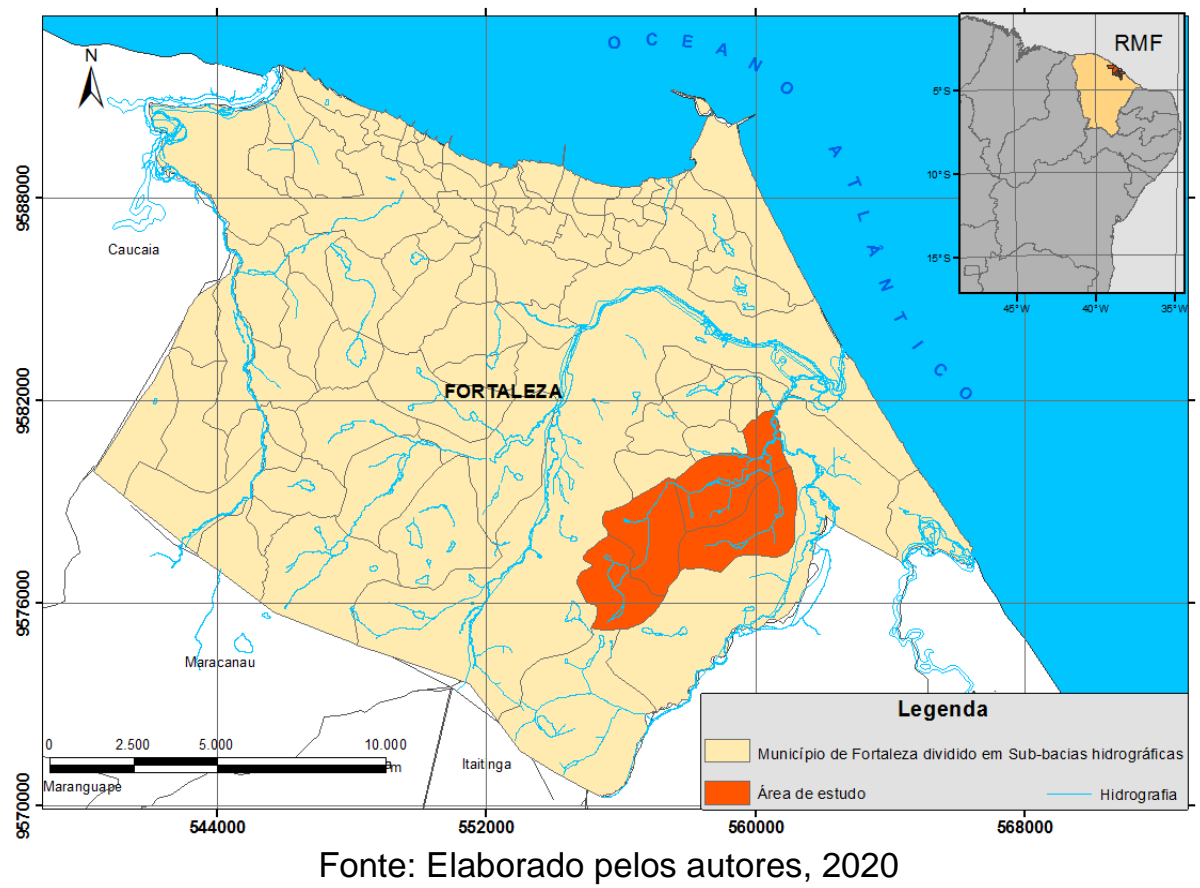

As metodologias de quantificação de áreas impermeáveis são de maneira comum estimadas por meio de densidades demográficas, como em Campana e Tucci (1994) que consideraram variáveis de densidades demográficas para dimensionamento de projetos de drenagem na cidade de São Paulo/SP. Esse tipo de metodologia pode resultar em erros de dimensionamento de vazão em projetos devido às diferenças de tipos de uso da terra, como comercial e industrial onde existe baixa quantidade de moradores.

Na busca de maior confiabilidade dos resultados, os estudos de impactos do uso e ocupação do solo no meio urbano vêm incorporando metodologias que aplicam ferramentas de geotecnologias, melhorando a qualidade eficiência da pesquisa. Principalmente, pelo poder de processamento de maior número de dados com maior rapidez e qualidade, envolvendo técnicas computacionais para estudos quantitativos e qualitativos (CAMPBEL e WYNNE, 2011).

Estudos têm sido realizados para determinar o fator da impermeabilização do solo, dentre eles na qualidade ambiental urbana, aplicando o geoprocessamento (LECHIU, 2012). Gutierrez et al., (2011), que utilizaram a classificação supervisionada manual onde as classes são distinguidas via vetorização pelo operador, em estudo temporal para classificação de imagens Landsat 5 - TM, que possui resolução espacial de $30 \mathrm{~m}$, na cidade de Campo Grande/MS. Já Reis (2011), aplicou esse mesmo método de classificação para as imagens IKONOS que tem $1 \mathrm{~m}$ de resolução especial na banda pancromática, para quantificar a impermeabilização na perspectiva intraurbana, comparando com valores de densidade demográfica numa sub-bacia hidrográfica na cidade de Santa Maria/RS. Estes estudos consideraram a importância de bacias hidrográficas urbanas para 0 
planejamento e gestão das cidades, visto a necessidade de gerir as águas urbanas para mitigação de problemas ambientais.

Piroli et al., (2012) utilizaram a mesma metodologia para analisar a transformação da paisagem urbana numa microbacia na cidade de Ourinhos/SP, classificando manualmente aerofotografias e imagem do satélite Quickbird de 0,6 m de resolução espacial. Pappas et al., (2011) realizaram experimento de simulação de diferentes intensidades de chuva e de percentuais de impermeabilização do solo, encontrando interferência da impermeabilização no aumento do regime de transporte de sedimentos. Piroli et al., (2012) e Pappas et al., (2011) demonstraram a relação entre o aumento do escoamento superficial (runnof) causado pela impermeabilização, e o consequente aumento da erosão do solo na área urbana.

Atualmente a principal aplicação de estudos de impermeabilização dos solos tem sido por meio da calibração de modelos hidrológicos para o desenvolvimento de projetos voltados à drenagem urbana. Nunes (2012) mensurou a impermeabilização do solo conforme os tipos de ocupação, utilizando a malha municipal em formato vetorial, interpretando e definindo as classes para o modelo hidrológico. Também para esse fim, Dans et al. (2013) definindo níveis de impermeabilização do solo por meio de imagem de altíssima resolução espacial IKONOS datada de 2003, com 1 metro de resolução na banda pancromátca, na qual foi realizada classificação manual em áreas estratégicas na Bacia Hidrográfica na cidade de Kleine/Bélgica, para calibração de imagens Landsat TM de 1982 e Landsat ETM+ de 2002, buscando avaliar o impacto da impermeabilização do solo na recarga dos aquíferos da bacia estudada entre os anos de 1982 e 2003.

Os estudos temporais do fenômeno de impermeabilização do solo, também possuem representatividade. Alves (2004) utilizou classificação pelo método do Vizinho Mais Próximo, atribuídos ao Normalization Difference Vegetation Index $N D V I$, de imagens brutas dos sensores CCD e HRC do satélite CBERS, e do sensor TM do satélite Landsat- 5 .

Kampouraki et al., (2008) aplicaram o método Geobject Based In Information Analysis (GEOBIA) na quantificação de áreas impermeáveis através de fotografias aéreas, realizando a segmentação das informações, a partir da transformação de diferentes propriedades nas imagens em geobjetos classificados por aerofotografias por meio do software eCognition.

Propõem-se, aqui, quantificar a taxa de ocupação do solo, ou seja, a porcentagem de área construída, que é proporcional ao percentual de impermeabilização, e comparar com os limites estabelecidos na lei orgânica 7.987/96 que trata do uso e ocupação do solo na cidade de Fortaleza. Os dados foram expressos em percentuais e agregados espacialmente por meio de polígonos correspondentes aos setores censitários da área. O estudo, portanto, desenvolve uma metodologia que pode ser aplicada em áreas urbanas para facilitar e agilizar a fiscalização, ajudando a promover maior efetividade de leis de uso e ocupação do solo urbano, no que tange a impermeabilização.

\section{MATERIAIS E MÉTODOS}

Tendo em vista a complexa realidade do espaço urbano, definida pela dinâmica das suas relações entre objetos a serem mapeados, há necessidade de imagem de detalhe espacial. Jensen (2009) coloca que quando se extrai informações urbanas/periurbanas de uso do solo, a partir de dados de sensores 
remotos, é importante ter resolução espacial, entre 0,25 e $5 \mathrm{~m}$, para a metodologia aplicada, quanto mais elevada resolução espacial dos dados, mais detalhada a informação que se pode extrair do ambiente urbano. Logo, foi escolhida a imagem Quickbird datada de 22 de junho de 2015, que possui uma resolução espacial de 2,8 $\mathrm{m}$ para as 4 bandas do VINIR (espectro eletromagnético do visível ao infravermelho próximo) e de 0,6 $\mathrm{m}$ para a banda pancromática. $\mathrm{O}$ uso das bandas espectrais da imagem Quickbird combinadas com a sua banda pancromática possibilita 0 melhoramento da resolução do produto, permitindo que os mapas sejam confeccionados na escala de 1:10.000 (Figura 2).

Figura 2. Localização e contexto de uso e cobertura da área

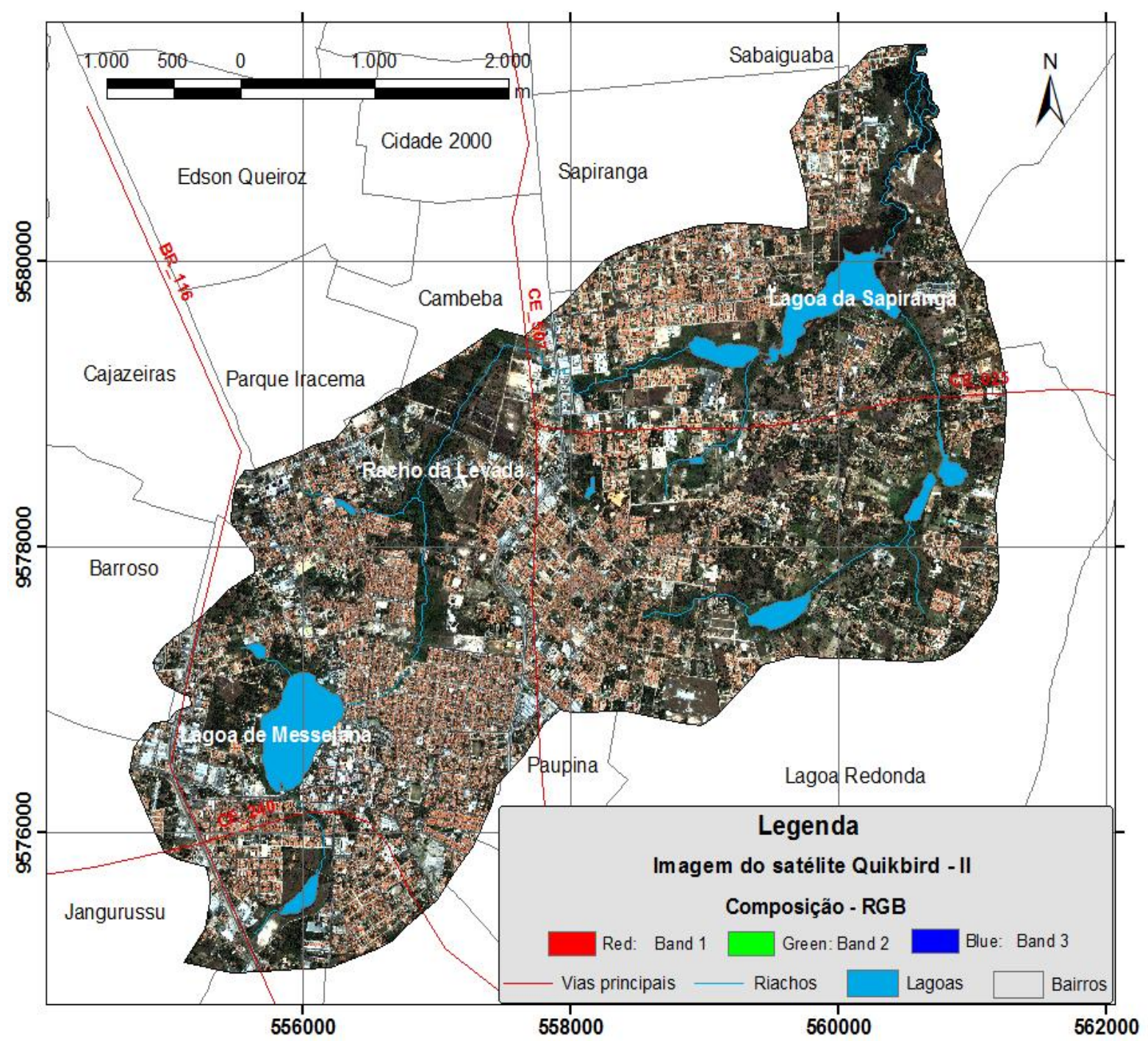

Fonte: Base cartográfica (IBGE, 2010); Imagem de satélite (SEMACE, 2015)

A execução do trabalho foi segmentada em 3 etapas (Figura 2). A Etapa 1 consistiu na aquisição das imagens Quickbird junto a Secretaria de Meio Ambiente do Ceará (SEMACE). As imagens estavam previamente compotas em formato RGB cor normal, portanto, devido a limitação dessa imagem, não foi possível realizar a composição com a banda do infravermelho próximo. Essas imagens consistem em 35 cenas em toda a área municipal, cerca de $313 \mathrm{~km}^{2}$, contudo foram utilizadas 2 cenas que abrangem a área de estudo que é de $20,9 \mathrm{~km}^{2}$. Depois de adquiridas, 
elas foram mosaicadas e georreferenciadas a partir de uma base cartográfica, pelo sistema de coordenadas UTM de projeção e utilizando o Datum SIRGAS 2000, o procedimento foi validado pelo levantamento em campo por meio de receptor Geographic Position System - GPS geodésico L1, com 12 pontos de controle. Por conseguinte, as imagens foram trabalhadas por meio de técnicas de Processamento Digital de Imagens (PDI) para realçar as informações superficiais com emprego da composição colorida, no sistema de canais de cores Red-Green-Blue (RGB), adicionada a banda pancromática no canal do Intensity (I) para melhorar a resolução espacial das imagens pela técnica de fusão RGBI. Logo a composição utilizada para análise das informações foi o $R(3) \mathrm{G}(2) \mathrm{B}(1) \mathrm{I}(\mathrm{P})$, que exibe uma imagem com as componentes do espectro do visível, possibilitando na identificação dos objetos numa análise visual.

$\mathrm{Na}$ Etapa 2 foi aplicada classificação das imagens pelo método GEOBIA (Figura 3). Se optou por esse tipo de classificação dado o avanço dessa técnica, sobretudo em áreas urbanas, além do modelo associado à lógica GEOBIA a qual se aproxima da percepção e interpretação humana (RADOUX et al., 2011). Tal método vem sendo muito utilizado em estudos intraurbanos, dado a sua melhor capacidade de extrair informações das imagens de altíssima resolução espacial, ou seja, imagens com resoluções espaciais menores que $1 \mathrm{~m}$ (MELO, 2002). O Termo GEOBIA evoluiu do conceito de Object Based in Informaion Analisis (OBIA), que foi um conceito que emergiu das chamadas GIScience. De acordo com Lang (2008, p. 6) "a principal proposta do OBIA é fornecer método automático de análise para imagens de altíssimas resoluções espaciais descrevendo as propriedades da imagem usando atributos espectrais, texturais, espaciais e topológicos"

Figura 3. Fluxograma da metodologia aplicada no trabalho.

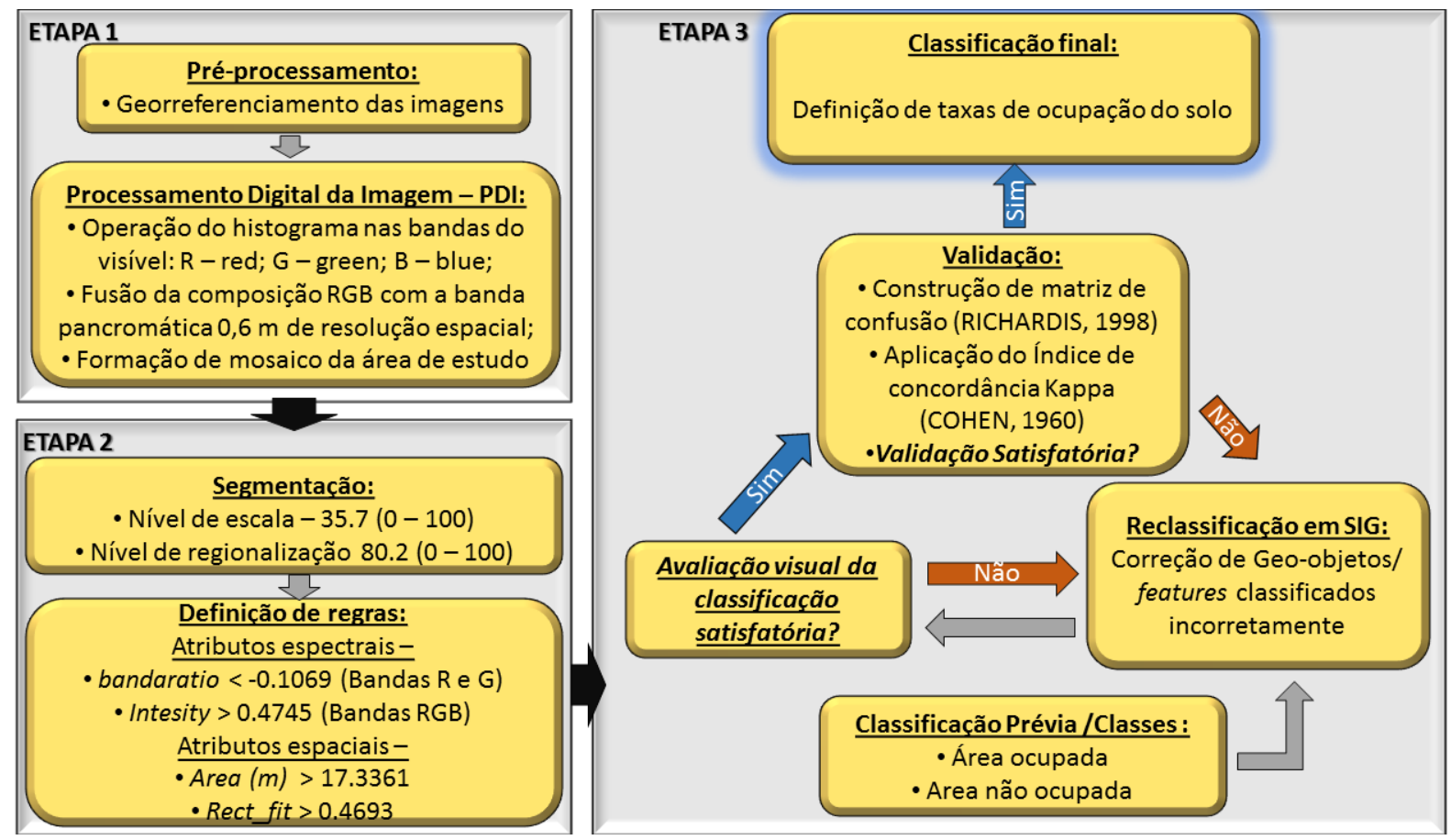

Fonte: Elaborado pelos autores, 2020

que difere esse método dos demais é a formação de objetos atribuídos à imagem conforme a escala trabalhada. Há, portanto a transformação da imagem em 
conjunto de objetos formados pela segmentação, e posteriormente agregados conforme a escala de regionalização, também chamada de merge (DE KOK, 2012). Para Hay e Castillo (2008) o GEOBIA foi o termo mais apropriado para aplicação em Geociências, pois os objetos estudados possuem o atributo geoespacial central, além de possibilitar a integração com conceitos geográficos e ecológicos quanto à diferenciação dos espaços. Os autores colocaram o GEOBIA como uma subdisciplina agregada as GISiences, que objetiva melhorar o processo de classificação automatizado ao aproximar este da interpretação e classificação manual.

O desenvolvimento de métodos automatizados reparte as imagens de sensoriamento remoto em significativas imagens-objetos, e avalia suas características através de escala. Este é o principal objetivo na geração de informações geográficas em formato para integração em ambiente SIG, no qual uma nova perspectiva é obtida (HAY e CASTILLO, 2008, p. 87).

Sua principal característica está na identificação das mudanças explicitas através da superfície imageada, em várias escalas, e a forma de gerenciá-las como objetos, relatando a complexidade da cena analisada, sendo também mais facilmente articuladas em ambiente SIG (KIM et al., 2011). A segmentação é uma metodologia crucial para o GEOBIA, mas não é exclusiva e nem muito diferente de outros procedimentos importantes para análise de geobjetos. Tal processo transforma a agregação ou agrupamento dos pixels vizinhos em médias, formando um objeto-imagem. Isso possibilita na distinção por contraste dos objetos formados em suas imediações. Foi assim aplicado segmentação dos geobjetos obedecendo aos critérios estabelecidos nas seguintes ordens (Quadro 1):

Quadro 1. Critérios para segmentação dos geobjetos

\begin{tabular}{l|l}
\hline Critérios & \multicolumn{1}{c}{ Definições } \\
\hline Critério 1 & $\begin{array}{l}\text { Teve-se como base o objetivo da classificação de residências cuja } \\
\text { proeminência no terreno se dá, sobretudo pelo telhado formado por } \\
\text { telhas de argila. }\end{array}$ \\
\hline Critério 2 & $\begin{array}{l}\text { Buscou-se um nível de segmentação que evitasse a fusão entre objetos } \\
\text { (oversegmantation), procurando minimizar os objetos que foram } \\
\text { posteriormente regionalizados. }\end{array}$ \\
\hline
\end{tabular}

Fonte: Elaborado pelos autores, 2020

Optou-se por segmentar a imagem tratada, utilizando o software ENVI.EX 4.5, em um nível de escala refinado em 37,4, numa amplitude de 0-100. Para realizar a regionalização dos objetos foi utilizado o valor 80,2, numa amplitude de 0 100, agrupando os objetos que correspondessem a área ocupada por residências O passo seguinte na classificação das imagens foi a distinção de dois tipos de objetos:

- Classe 1 (Área ocupada) - corresponde a construção residencial, haja vista a limitação espectral inerente à imagem optou-se por extrair apenas essa classe. A imagem foi composta em formato RGB cor real, assim como não se dispõe de bandas do infravermelho, o seu principal atributo é a altíssima resolução espacial, que possibilitou extrair a classe: área ocupada. 
- Classe 2 (Área não ocupada) - corresponde a todos os objetos não identificados como área ocupada.

Tais critérios foram escolhidos tendo como foco o objetivo do estudo, identificando os objetos por atributos espaciais e espectrais dispostos em regras que foram pré-estabelecidas segundo as características espectrais e espaciais dos representativas dos objetos.

Os atributos espectrais aplicados para análise dos geobjetos por meio do software ENVI. EX 4.5 (Tabela 1) correspondem o Bandratio e o Intensity. 0 Bandratio é um tipo de classificador que apresenta a resultante da diferença normalizada entre duas bandas. Para esta aplicação foram escolhidos os canais $1 \mathrm{e}$ 2 , verde e vermelho, respectivamente. Este processamento conseguiu separar objetos considerados como cobertura vegetal. Por sua vez o Intensity separa as diferentes intensidades de brilho entre os objetos, por exemplo, solo exposto possui alta reflectância e o asfalto apresenta baixa reflectância. Já os atributos espaciais aplicados foram a Área e o Rect_fit. O primeiro tratou da definição mínima dos geobjetos classificados e o segundo calculou o quão próximo o objeto é de um retângulo, sendo eficiente para subtrair formas irregulares da classe almejada.

Tabela 1. Regras para atributos aplicados na classificação

\begin{tabular}{c|c|c|c|c}
\hline \multirow{2}{*}{ Propriedades } & \multicolumn{2}{|c|}{ Atributo Espectral } & \multicolumn{2}{c}{ Atributo Espacial } \\
\cline { 2 - 5 } & Bandratio & Intensity & Área & Rect_fit \\
\hline Intervalo de valores & $-1,0-1,0$ & $0-1,0$ & - & $0-1$ \\
\hline $\begin{array}{c}\text { Regras para } \\
\text { classificação }\end{array}$ & $<-0,1059$ & $>0,4745$ & $>17,3361$ & $>0,4693$ \\
\hline
\end{tabular}

Fonte: Elaborado pelos autores, 2020

As dificuldades que envolveram a classificação das imagens se encontram na limitação da resolução radiométrica da imagem de 11 bits, sendo, frequentemente insuficientes para realizar boa classificação em imagens de altíssima resolução, além da diversidade e quantidade de objetos que podem ser diferenciados no espaço urbano, e complexidade de os diferenciar (MENESES e ALMEIDA, 2012).

Ao se aplicar os filtros nas regras determinadas anteriormente, houve, na avaliação das regras relativas ao atributo espectral, uma boa extração dos objetos correspondente as residências com telhados de assinatura espectral característicos de telhas de argila. No entanto, havia residências, ocupações industriais e comerciais que possuíam assinaturas espectrais do telhado fora do padrão investigado, isso porque utilizavam materiais distintos como por exemplo cobertura das construções usando telhado de zinco. Foi, então, realizada uma reclassificação da imagem de maneira manual em ambiente SIG - Arcgis 10.2.2, onde foram incorporados parte das residências não classificadas, além das construções não residenciais, como indústrias e comércios e áreas de estacionamento. $O$ asfalto não foi agregado a área ocupada, sendo incorporado após a classificação em índice proposto pela Lei Municipal no 7.987/96. A junção de elementos de diferentes respostas espectrais se deu, especialmente, por meio do treinamento dos índices espaciais. O GEOBIA permitiu classificar de forma eficiente esses elementos por seus atributos espaciais com as ferramentas de tamanho da área e rect_fit.

A reclassificação consistiu na conversão da primeira imagem gerada pela classificação GEOBIA em arquivo vetorial no SIG, neste caso no formato shapefile. 
No processo seguinte, foi realizada validação da classificação por meio da tabela de confusão. Para o processo de validação houve campanhas de campo para identificação da verdade terrestre. Comparando pontos classificados com relação ao modelo aplicado e a realidade que esse modelo buscou representar. A matriz de confusão consiste na identificação de pontos, representados por pixels dentro da imagem, estes foram avaliados segundo a verdade terrestre, e classificados como corretos ou incorretos. Na tabela aplicada, buscou-se avaliar os erros de inclusão, os quais correspondem ao erro total, já que a classificação consiste em 2 classes: área ocupada, área não ocupada.

Foi utilizado um número de amostras de 300 pontos, os quais foram avaliados e dispostos na matriz de confusão, para a avaliação dos erros de inclusão, ou seja, quando o ponto pertence a uma classe diferente da classe classificada. A exatidão global consiste na identificação média de acertos para cada classe (Tabela 2).

Tabela 2. Matriz de confusão

\begin{tabular}{|c|c|c|c|c|c|c|}
\hline \multirow{2}{*}{\multicolumn{2}{|c|}{$\begin{array}{c}\text { Comparação } \\
\text { Modelo vs } \\
\text { Verdade }\end{array}$}} & \multicolumn{2}{|c|}{ Verdade de campo } & \multirow{3}{*}{$\begin{array}{c}\begin{array}{c}\text { Total de } \\
\text { Pixels }\end{array} \\
51\end{array}$} & \multirow{3}{*}{$\begin{array}{c}\begin{array}{c}\text { Erros de } \\
\text { Inclusão } \\
(\%)\end{array} \\
17,65\end{array}$} & \multirow{3}{*}{$\begin{array}{c}\begin{array}{c}\text { Pixels bem } \\
\text { classificados } \\
\text { (\%) }\end{array} \\
82,35\end{array}$} \\
\hline & & \multirow{2}{*}{$\begin{array}{c}\begin{array}{c}\text { Área } \\
\text { ocupada }\end{array} \\
42\end{array}$} & \multirow{2}{*}{\begin{tabular}{|c|}
$\begin{array}{c}\text { Área } \\
\text { não } \\
\text { ocupada }\end{array}$ \\
9
\end{tabular}} & & & \\
\hline 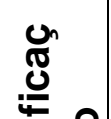 & $\begin{array}{c}\text { Área } \\
\text { ocupada }\end{array}$ & & & & & \\
\hline $\begin{array}{l}\mathscr{D}^{n} \mathbf{2 \pi} \\
\frac{\pi}{0} \\
0\end{array}$ & $\begin{array}{l}\text { Área não } \\
\text { ocupada }\end{array}$ & 9 & 240 & 249 & 3,62 & 96,38 \\
\hline \multicolumn{2}{|c|}{ Total de pixels } & 51 & 249 & 300 & $\begin{array}{c}\text { Exatidão } \\
\text { Global }\end{array}$ & $94 \%$ \\
\hline
\end{tabular}

Fonte: adaptada de Richards (1998)

Por meio da matriz de confusão, foi composto o coeficiente de concordância Kappa, utilizado por Cohen (1960) para validação da classificação, este coeficiente é um dos mais utilizados para a função, pois proporcionam que todos os elementos da matriz são considerados, erros de omissão, erros de inclusão, assim como o percentual de pixels bem classificados e total de pixels analisados. O coeficiente de Kappa é composto pela Equação (1):

$$
\mathrm{K}=\frac{N \sum_{\mathrm{i}=1}^{\mathrm{p}} x i i-\sum_{\mathrm{i}=1}^{\mathrm{p}} x i}{N^{2}-\sum_{\mathrm{i}=1}^{\mathrm{p}} x i}
$$

Onde: $\Sigma$ xii $=$ Somatório de pixels classificados corretamente; $\Sigma$ xi $=$ Somatório de pixels classificados incorretamente; $\mathrm{N}=$ Número total de pixels; $r=$ Número de linhas e colunas.

$\mathrm{Na}$ área estudada há o limite de taxa de ocupação de $45 \%$, e limite de $60 \%$ de área impermeável. Denota-se, portanto, que para a implantação da Lei ํo 7.987/96, foi fixado que $15 \%$ da impermeabilização pela pavimentação asfáltica. Foi considerado esse valor como fixo adicionado à taxa de ocupação, identificando assim a impermeabilização do solo. 15\% também é considerado por Tucci (2006) como a percentagem de impermeabilização causada por vias asfaltadas. 
As taxa de ocupação foram calculados conforme a (Equação 2):

$$
\mathrm{TI}=\mathrm{To}+15
$$

Onde: $\mathrm{Tl}=$ Taxa de impermeabilização do solo; To = Taxa de Ocupação; e 15\% é o valor em porcentagem da taxa de impermeabilização média na área, causada pelas vias asfaltadas, segundo Lei ํㅜ 7.987/96.

A quantificação das taxas de ocupação foi definida por setor censitário, dado seu atributo de escala que possibilitou melhor espacialização do fenômeno estudado. A classificação foi validada pelo coeficiente de conformidade de Kappa, que calculou o valor de 0.78 portanto, há conformidade substancial, de acordo com Landis e Koch (1977), (Tabela 3).

Tabela 3. Valores de Índice de conformidade Kappa.

\begin{tabular}{|c|l|}
\hline $\begin{array}{c}\text { Valores de } \\
\text { Kappa }\end{array}$ & \multicolumn{1}{c|}{ Interpretação } \\
\hline$<0$ & Sem conformidade \\
\hline $0-0.19$ & Pouca conformidade \\
\hline $0.20-0.39$ & Média conformidade \\
\hline $0.40-0.59$ & Moderada conformidade \\
\hline $0.60-0.79$ & Conformidade substancial \\
\hline $0.80-1.00$ & Conformidade quase perfeita \\
\hline \multicolumn{2}{|c|}{ Fonte: Landis e Koch, (1977) } \\
\hline
\end{tabular}

Os dados foram compilados em setores censitários, para melhor representação espacial na área de estudo. Também foram comparadas às taxas de ocupação máximas permitidas.

\section{RESULTADOS E DISCUSSÃO}

A lei orgânica o $7.987 / 96$ considera as Macrozonas de Densidade classificadas como: Macrozona Urbanizada, a área atendida integralmente pela rede de abastecimento de água e parcialmente pela rede de esgotos e com projetos de expansão desta, nessa zona se verifica maior concentração da população e melhores condições de infraestrutura básica; a Macrozona Adensável é a área atendida em parte pelo sistema de abastecimento d'água, sem sistema de coleta de esgotos, onde se verifica uma tendência de expansão das atividades urbanas; a Macrozona de Transição é a área do município não adensada, sem infraestrutura de água e esgotos, configurando-se como áreas de reservas para a expansão urbana; as Macrozonas Especiais, por sua vez, são identificadas por características ambientais, culturais e econômicas, que exigem condições diferenciadas de ocupação ou o não aproveitamento direto dessas áreas para tal, isso por conta dos riscos associados às condições naturais ou mesmo a preservação do patrimônio histórico e cultural.

Essas macrozonas estão diferenciadas também com relação aos índices máximos de ocupação e de impermeabilização do solo, os quais estão estabelecidos na referida lei (Tabela 4). No caso da Macrozona Urbanizada existem subdivisões 
que exprimem especificidade para determinadas áreas da cidade as quais são classificadas como zonas.

Tabela 4. Macrozonas de Densidade

\begin{tabular}{l|c|c}
\hline $\begin{array}{c}\text { Macrozonas de } \\
\text { Densidade }\end{array}$ & Permeabilidade \% & $\begin{array}{l}\text { Taxa de } \\
\text { ocupação \% }\end{array}$ \\
\hline Macrozona Urbanizada & $20-40^{*}$ & $45-60^{*}$ \\
\hline Macrozona Adensável & 40 & 45 \\
\hline Macrozona de Transição & 40 & 45 \\
\hline Macrozonas Especiais & $10-50$ & $80-33$ \\
\hline \multicolumn{2}{r}{ Fonte: } & Lei orgânica no $7.987 / 96$
\end{tabular}

* Variam conforme as subdivisões das zonas estabelecidas no plano

A área abordada nesse estudo é parcelada no plano diretor como áreas com processos de ocupação relativamente menores. Inserindo-se, desse modo, em Macrozona Adensável e Macrozona de Transição (Figura 5).

Figura 5. Macrozonas de Densidade

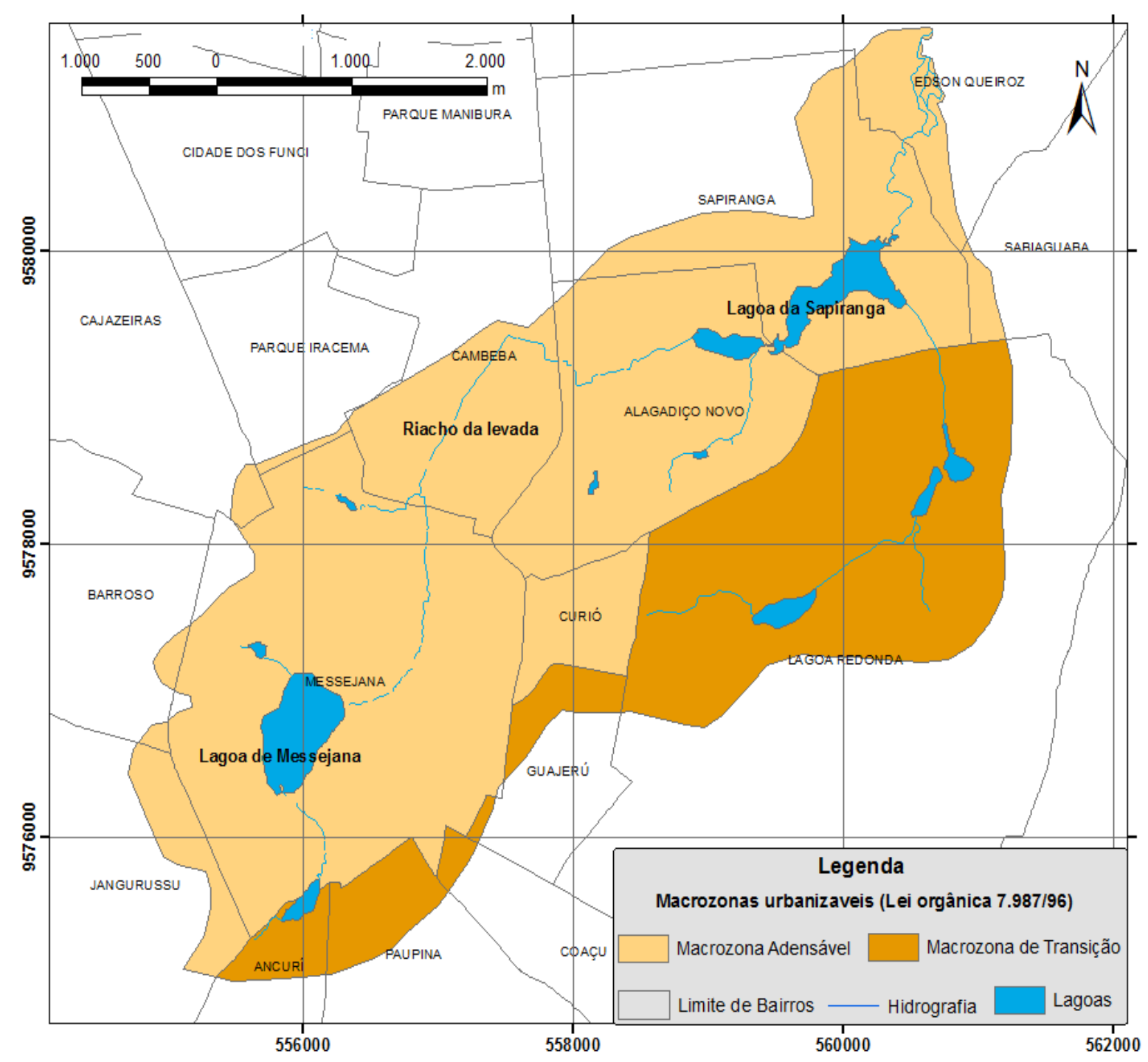

Fonte: Autores, adaptada de Lei orgânica n. 7987/96 
Os valores atribuídos, conforme a Tabela 5, a cada macrozona de densidade é congruente com o planejamento municipal em torno da expansão urbana da cidade. O planejamento é orientado a buscar a compatibilização de uso da terra conforme as potencialidades e limitações dos sistemas ambientais e socioeconômica deste, tanto em decorrência das condições naturais, como dos serviços, empreendimentos e equipamentos urbanos. De modo que, de acordo com alguns dos objetivos do plano, constado no Art. 1을

A ordenação das funções da cidade através da utilização racional do território, dos recursos naturais, e do uso dos sistemas viário e de transporte, quando do parcelamento do solo, da implantação e do funcionamento das atividades industriais, comerciais, residenciais e de serviços; II - a preservação e a proteção do ambiente natural e cultural; IV - a compatibilidade da densidade das atividades urbanas com as condições naturais, bem como com a infraestrutura instalada e projetada; $\mathbf{V}$ - a intensificação do processo de ocupação do solo, à medida que houver ampliação da capacidade da infraestrutura preservando-se a qualidade de vida da coletividade.

A taxa limite de impermeabilização do solo é uma importante medida pois, ela é diretamente ligada à taxa de ocupação e é indicada para diminuir o impacto da urbanização no aumento do escoamento superficial e consequentemente na maior frequência e magnitude das inundações.

Áreas ocupadas foram extraídas como classe a ser interpretada como impermeabilização, conforme mapa (a) (Figura 6). Os dados de taxa de ocupação compilados em setores censitários permitiram melhor representação espacial, de modo a comparar as condições atuais de ocupação com os valores limites estabelecidos por lei. Além disso, pode-se observar a relação das áreas ocupadas com os aglomerados subnormais definidas pelo IBGE (2010), os quais definem áreas sem regularidade urbanística (Figura 7).

Figura 6. Classe áreas impermeabilizadas pela urbanização

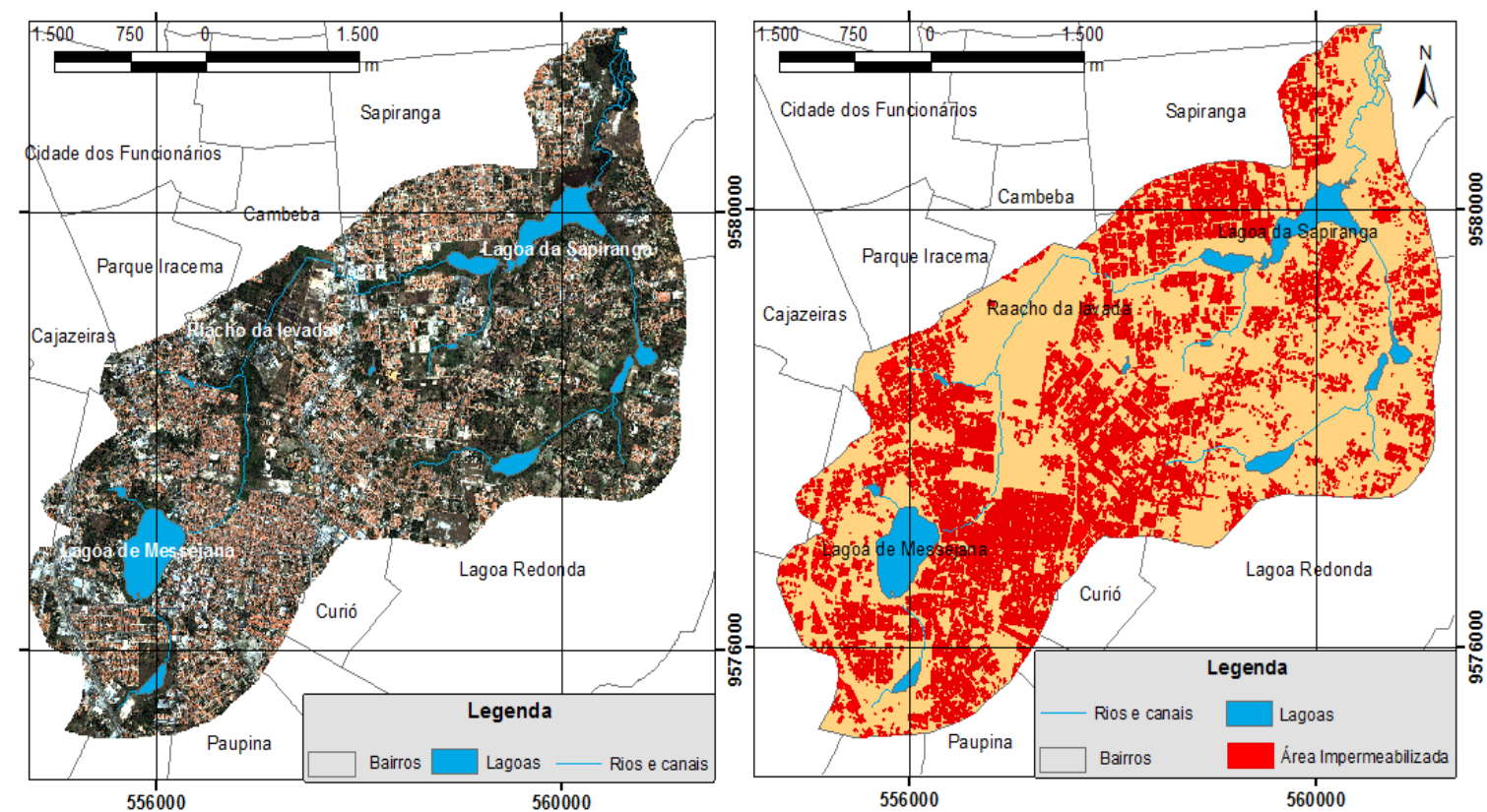

Fonte: Elaborado pelos autores, 2020 
A área analisada possui 107 setores censitários, no entanto, alguns não são representativos, pois, se encontram em pequena parcela dentro da área de estudo, assim setores com menos de 30\% de sua área dentro da área de estudo não foram considerados pois grande parte dados aprendidos estariam fora da área de estudo. Portanto, em 93 unidades foram calculadas as taxas de ocupação e impermeabilização e observou-se que alguns setores censitários de maior taxa de ocupação são aglomerados subnormais.

Figura 7. Distribuição taxas de ocupação por setores censitários

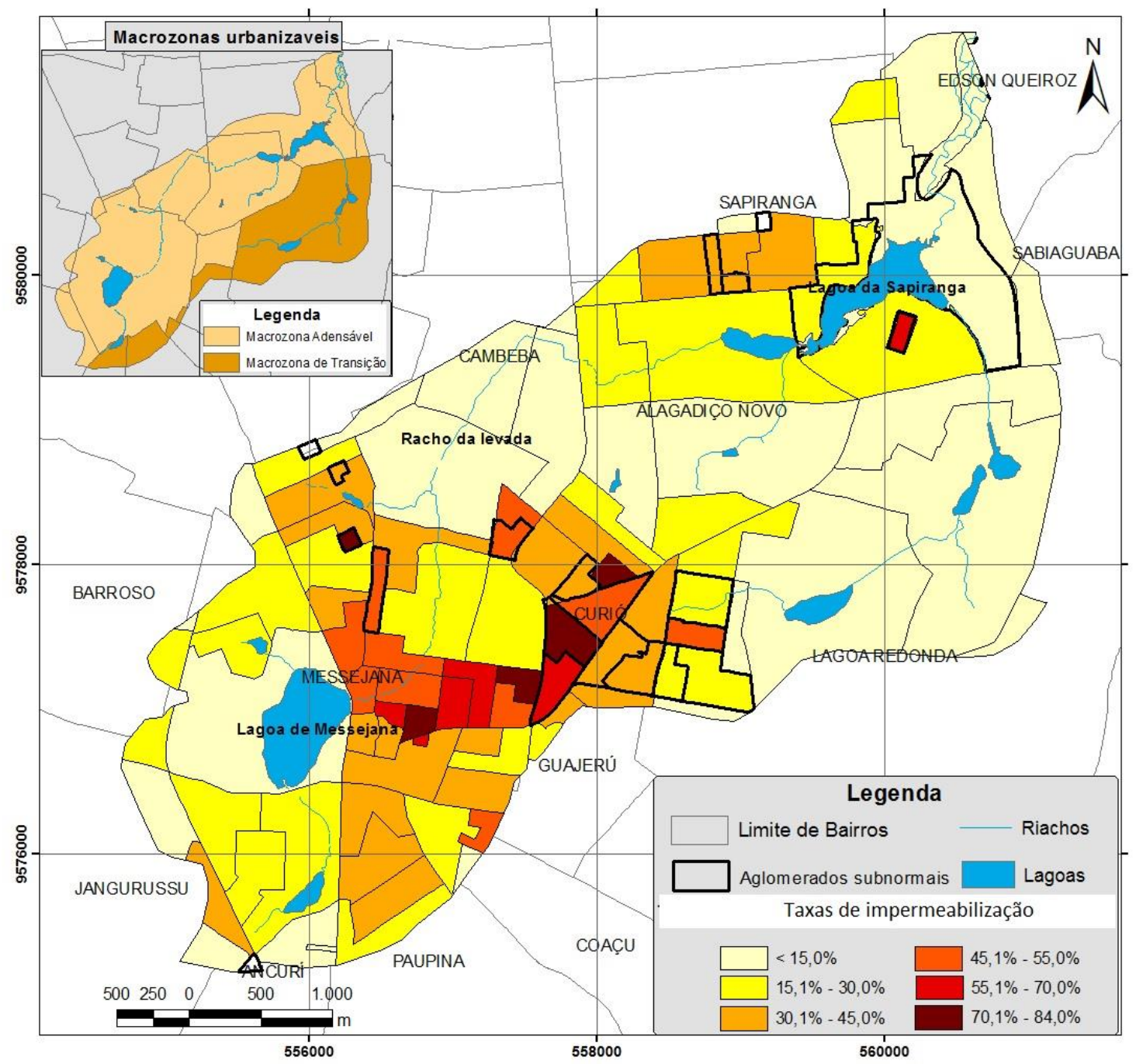

Fonte: Elaborado pelos autores, 2020

Os setores censitários com menores áreas possuem altas taxas de ocupação, pois na escolha de seus critérios genéticos pelo IBGE a quantidade de população é um relevante atributo a considerar. Identifica-se, de acordo com a legislação municipal que parte dos setores estudados não se encontra em conformidade legal (Tabela 5). Como a classificação realizada não considerou as vias asfálticas que ocorrem em toda a área, a impermeabilização causada por elas foi adotada em $15 \%$ conforme recomenda Tucci (2006). Assim além da proporção da taxa de ocupação aqui calculada, foi adicionado mais $15 \%$ relativo à impermeabilização por asfalto. 
Constatou-se que 22 setores estão fora dos padrões estabelecidos pela Lei 7.987/96. Apresentando níveis de impermeabilização entre 60 e $85 \%$, mesmo após vários anos após a regulamentação dessa lei. Nos locais onde os valores de impermeabilização do solo são mais acentuados há maior potencial para que ocorra escoamento superficial e isso pode provocar maior frequência e magnitude de inundações. Leopold (1968) afirma que a taxa de escoamento superficial pode aumentar cerca de 6 vezes os valores de vazão média de pré-desenvolvimento 6 . Gutiérrez et al., (2011) avalia que o crescimento das áreas impermeáveis pode causar cerca de $80 \%$ de escoamento superficial em uma chuva de $100 \mathrm{~mm}$.

Tabela 5. Classes de ocorrência da impermeabilização dos setores censitários

\begin{tabular}{c|c|c|c|c}
\hline $\begin{array}{c}\text { Taxas } \\
\text { de } \\
\text { Ocupaçã } \\
\mathbf{0}(\%)\end{array}$ & $\begin{array}{c}\text { Área média } \\
\text { imperm. } \\
\text { por vias } \\
\text { asfálticas* } \\
(\%)\end{array}$ & $\begin{array}{c}\text { Área } \\
\text { imperm. } \\
(\%)\end{array}$ & $\begin{array}{c}\text { Valor } \\
\text { Máximo de } \\
\text { área } \\
\text { imperm.* (\%) }\end{array}$ & $\begin{array}{c}\text { Frequência de } \\
\text { Setores censitários } \\
\text { por classe }\end{array}$ \\
\hline $0 \geq 15$ & 15 & $15 \geq 30$ & 60 & 18 \\
\hline $15 \geq 30$ & 15 & $30 \geq 45$ & 60 & 26 \\
\hline $30 \geq 45$ & 15 & $45 \geq 60$ & 60 & 24 \\
\hline $45 \geq 55$ & 15 & $60 \geq 70$ & 60 & 4 \\
\hline $55 \geq 70$ & 15 & $70 \geq 85$ & 60 & 4 \\
\hline $70>$ & 15 & $85>$ & 60 & 93 \\
\hline \multicolumn{4}{c}{$\begin{array}{c}\text { Fonte: Elaborado pelos autores, 2020 } \\
\text { Obs.: imperm. = impermeabilizada. }\end{array}$} \\
\hline \multicolumn{5}{c}{ * Valores estabelecidos pela Lei orgânica № 7.987/96. }
\end{tabular}

As áreas de maior impermeabilização não necessariamente estão sujeitas a inundações, visto que, existem outros fatores que influenciam nessa dinâmica. Apesar das áreas em ocupação serem relacionadas ao processo de impermeabilização, compactação do solo e consequente produção de escoamento superficial (VAZEA, et al, 2010), há fatores naturais que estão ligados aos riscos de inundações, como a geomorfologia da área e tipo de drenagem, tipos de solo, litologia e proximidade de níveis de base de erosão, representados por lagoas, rios canais (SOUZA et al., 2012).

Foram observadas áreas em que a taxa de ocupação e impermeabilização ultrapassam o estabelecido em lei. De modo que, as políticas de aproveitamento da terra são mal implantadas podendo resultar em sérios danos socioambientais. Essa condição é dependente do risco ambiental associado aos fatores naturais, como também da vulnerabilidade social inerente à população que ocupa essas áreas (ZANELLA, et al., 2013). De um modo geral o aumento da impermeabilização do solo é constatado de maneira a acompanhar a expansão horizontal da cidade de Fortaleza, associado muitas vezes a aglomerados subnormais com elevados índices de ocupação e, consequentemente, de impermeabilização do solo. De acordo com Tucci (2007) o primeiro passo da gestão do espaço urbano é recuperar a

\footnotetext{
${ }^{6}$ Segundo Tucci (2007) se refere a vazão de pré-desenvolvimento como a vazão que ocorrerem anteriormente ao processo de urbanização/ocupação urbana de uma bacia hidráulica.
} 
capacidade de gerenciamento da cidade por parte do poder público de maneira a promover e/ou recuperar a capacidade de amortecimento inerente ao processo de infiltração.

\title{
CONCLUSÃO
}

Os planos de uso e ocupação do solo são essenciais para o planejamento e gestão urbana. Eles podem implementar medidas não estruturais, como zoneamentos baseadas em medidas restritivas de uso do solo, que são importantes em minimizar os impactos sobre os sistemas ambientais locais, sobretudo quanto a dinâmica hidroclimática, uma vez que, isso pode reduzir substancialmente as inundações urbanas, diminuindo a necessidade de medidas estruturais de drenagem, que consomem enormes quantidades de recursos financeiros públicos.

Torna-se imperativo que esses planos promovam resultados efetivos na ocupação do solo urbano, para isso é necessário quantificar e avaliar seus resultados. Buscando isso, a construção da metodologia, aqui desenvolvida e aplicada, envolveu integração do sensoriamento remoto na aquisição, e processamento e classificação de imagens de satélite com o manejo de dados no SIG, possibilitando resultado satisfatório, dentro das limitações espectrais e radiométricos da imagem.

O geoprocessamento, enquanto metodologia analítica, pode ser aplicado na mensuração da impermeabilização e da ocupação do solo em áreas urbanas. A agregação desses resultados em setores censitários pode ser utilizada no processo fiscalização, pois, as ações in lócus podem se concentrar nos setores que apresentam áreas com maiores taxas de impermeabilização e ocupação do solo, promovendo a maximização do uso de recursos públicos disponíveis para fiscalização.

Constatou-se que na área de estudo, em 2015, a ocorrência de aglomerados subnormais, onde existem altas taxa de ocupação, no entanto, o excesso de impermeabilização não é exclusivo desses setores, mas também em certas parcelas ocupadas por padrões urbanísticos "normais" que intensificam a ocupação do lote. Foi observado que 22 setores estão fora dos padrões estabelecidos pela Lei 7.987/96, apresentando níveis de impermeabilização entre 60 e 85 \%.

Os resultados apontaram núcleos com maiores taxas de ocupação e impermeabilização, sendo essas as áreas prioritárias, que necessitam de ações de fiscalização mais eficazes.

O planejamento e gestão urbana dependem da sua efetividade, as medidas restritivas de uso do solo, precisam ser instrumentalizadas com base em inteligência geográfica no seu processo de identificação e avaliação de áreas prioritárias, gerando banco de dados e informações geográficas para subsidiar políticas públicas urbanas mais sustentáveis.

\section{REFERÊNCIAS BIBLIOGRÁFICAS}

\begin{abstract}
ALVES, C. A. Estimativa da Área impermeável dentro da Bacia Hidrográfica do Arroio Dilúvio (Porto Alegre/RS) Através de Técnicas de Sensoriamento Remoto e Geoprocessamento. (Mestrado em Geografia). Centro Estadual de Pesquisas em Sensoriamento Remoto e Meteorologia - CEPSRM, Universidade Federal do Rio Grande do Sul. Porto Alegre, 2004.
\end{abstract}


CAMPANA, N. A., TUCCI, C. E. M. Estimativa de área impermeável de macrobacias urbanas: RBE - Caderno de Recursos Hídricos. v. 12 , n. 2 : 79-94. 1994.

CAMPBEL, J. B.; WYNNE. R. H. Introduction to Remote Sensing. 5. ed. New York: The Gluilford Press, 2014.

CANHOLI, A. P. Drenagem urbana e controle de enchentes. 2. ed. São Paulo: Oficina de textos, 2014.

COHEN, J. A Coeficiente of Agreement for Nominal Scales. Education and Psycological Measurement. v. 20 n. 1: p. $37-43,1960$.

DANS, J.; DUJARDIN, J.; REGGERS, R.; BASHIR, I.; CANTERS, F.; BATELAAN, O. Mapping impervious surface change from remote sensing for hydrological modeling. Journal of Hydrology, v. 485: p. 84-95. 2013, doi: https://doi.org/10.1016/j.jhydrol.2012.09.045

DE KOK, R. Spectral Diference in the Image Domain for Large Neighborhoods, a GEOBIA Pre-Processing Step for High Resolution Imagery. Remote Sensing. v. 4 n. 8: p. 2294 - 2313. 2012. doi: 10.3390/rs4082294

FORTALEZA, Prefeitura Municipal de Fortaleza. Lei de Uso e Ocupação do solo. Lei №. 7.987 de 23 de dezembro de 1996.

GUTIERREZ, L. A. R.; SOUZA, G. F.; PEREIRA, G.; PARANHOS FILHO, A. C.; ARIMA, G. A.; BARBASSA, A. P. Mapeamento Temporal dos Índices: Área de superfície impermeável e Escoamento superficial da Area Urbanizada de Campo Grande/MS. Caminhos de Geografia. Uberlândia, v.12, n. 37, p. 269-288, 2011.

HAY, J.; CASTILLA, G. Geographic Object-Based Analysis (GEOBIA): a new name for a new discipline. In: Blasthke, T.; Lang, S.; J. Hay. (Orgs). Object-Based Image Analysis: spatial concept for knowledge driven remote sensing application. Berlin: Springer, p. $3-28.2008$.

JENSEN, J. R. Sensoriamento Remoto do Ambiente: uma perspectiva em recursos terrestres. 2. ed.; tradução José Carlos Neves Epiphanio (Coord.) et al. São José dos Campos, SP: Parêntese. 2009.

KAMPOURAKI, M.; WOOD, G. A.; BREWER, T. R. Opportunities and limitations of object-based image analysis for detecting urban impervious and vegetated surfaces using true-colour aerial photography. In: Blasthke, T.; Lang. S.; Hay, J. (Org's). Object-Based Image Analysis: spatial concept for knowledge driven remote sensing application. Berlin: Springer, p. 555-570, 2008.

KIM, M.; WARNER, T. A.; MADDEN, M.; ATKINSON, D. S. Multi-scale GEOBIS with very high spatial resolution digital aerial imagery: scale, texture and image objects. International Journal of Remote Sensing. v. 32, n.10, p. 2825 - 2850, 2011 Doi https://doi.org/10.1080/01431161003745608 
LANG, S. Object Based Analysis for remote sensing applications: modeling reality dealing with complexity. In: Blasthke, T.; Lang, S.; Hay, J. (Orgs). Object-Based Image Analysis: spatial concept for knowledge driven remote sensing application. Berlin: Springer, p. 3 - 28. 2008.

LANDIS, J.; KOCH, G. The measurement of observer agreement for categorical data. Biometrics. n. 33: p. 159-174. 1977.

LECHIU, B. C. G.; OLIVEIRA FILHO, P. C. SOUSA, J. B. Utilização de imagens orbitais de alta resolução em superfícies com níveis distintos de impermeabilização do solo: caso de Irati - PR. Semina: Ciência e Tecnologia, v. 33 n. 2, p.127 - 140. 2012, doi:10.5433/1679-0375.2012v33n2p127

LEOPOLD, L. B. Hydrology for urban planning - a guide book on the hydrologic effects on urban land use. 554. New York, United States Geology Survey. 1968.

MATTES, A. O problema da drenagem urbana. In: Dowbor, L.; Tagnin, R. A. Administrando a água como se fosse importante: gestão ambiental e sustentabilidade. 1. Ed, São Paulo: Editora SENAC, p. 14 - 20, 2005.

MELO, D. H. C. T. B. Uso de dados Ikonos II na análise urbana: testes operacionais na zona leste de São Paulo. Dissertação (Mestrado). São José dos Campos: Instituto Nacional de Pesquisas Espaciais, 2002.

MENESES, P. R.; ALMEIDA, T. Introdução ao Processamento de Imagens de Sensoriamento Remoto. Brasília: UnB/CNP. 2012.

NUNES, F. G.; FIORI, A. P. (2008) Análise da Impermeabilização dos Solos e Aumento dos Picos de Vazão de Cheias em Bacias Hidrográficas Urbanas. Boletim Paranaense de Geociências, n. 62-63, p.47-60. 2008

PAPPAS, E. A.; HUANG, C.; MASCE, J. V. B. Do Upslope Impervious Surfaces Impact the Run-on/Runoff Relationship? Jornal of Hidrologic Engeneering. n.16: $p$. 345-350, 2011.

PIROLI, E. L.; PERUSI, M. C.; ZANATA, J. M. Mudança no Uso da Terra e Impacto sobre o Solo da Microbacia Hidrográfica do Córrego Água da Veada, Ourinhos/SP. Revista Geonorte, Edição Especial, v.1 n. 4, p. 855-865, 2012.

RADOUX, J.; BOGAERT, P. FASBENDER, D.; DEFOURNY, P. Thematic acuracy assessment of geographic object-based image classification., International Jornal of Geographical Information Science, v. 25 n. 6. 895 - 911, 2011.

REIS, J. T.; PEREIRA FILHO, W.; SILVEIRA, A. L. L. Estimativa entre densidade habitacional e áreas impermeáveis na região urbana da sub-bacia hidrográfica do arrio Cadena em Santa Maria, Ciência e Natureza, UFSM, v.1, n. 33, p. 145-160, 2011. Doi http://dx.doi.org/10.5902/2179460X9983.

RICHARDS, J. A.; JIA, X. Remote Sensing Digital Image Analysis. Springer. 3 ed edition. Canberra. 1998. 
SOUZA, C. F.; CRUZ, M. A.; TUCCI, C. E. M. Desenvolvimento Urbano de Baixo Impacto: Planejamento e Tecnologias Verdes para a Sustentabilidade das Águas Urbanas, Revista Brasileira de Recursos Hídricos, v.17, n. 2, p. 9-18. 2012.

TUCCI, C. E. M. Água no meio Urbano. In: Rebouças, A. C.; Braga, B. Tundisi, J. G. Águas Doces no Brasil: capital ecológico, uso e conservação. 3. ed. São Paulo: Escrituras: p. 399-432. 2006.

TUCCI, C. E. M. Gestión de Inundaciones Urbanas. Porto Alegre: Editora Evangraf Ltda. 2007.

VAZEA, R. F.; OLIVEIRA FILHO, P. C.; MAIA, A. G.; DISPERATI, A. A. Uso e ocupação do solo em bacia Hidrográfica Urbana a partir de Imagem Orbitais de Alta Resolução. Floresta e Ambiente, v.17, n. 1, p. 23 -29. 2010.

ZANELLA, M. E.; OLÍMPIO, J. L.; COSTA, M. C. L.; DANTAS, E. W. C. (Vulnerabilidade Socioambiental do Baixo Curso da Bacia Hidrográfica do Rio Cocó. Sociedade \& Natureza: Uberlândia, v. 25, n. 2, p. 317 - 332. 2013. 